\title{
Quellen- und Literaturverzeichnis
}

\section{Quellenverzeichnis}

\author{
Ungedruckte Quellen
}

Kurze Bestandsbeschreibung:

Der relevante Bestand im Bundesarchiv setzt sich zum einen aus der umfangreichen Überlieferung des Reichsversicherungsamtes zusammen. Hier sind vor allem drei Teilbestände betr. RfA wichtig: Erstens 20 Bände zu „Aufsicht über die RfA“ (R 89/3387-3467), 23 Bände zu „Personalangelegenheiten RfA“ (R 89/3478-3499) sowie neun Bände „Personalangelegenheiten RfA/Einzelfälle“ (R 89/3500-3508) und eine Reihe von Kartons mit hunderten von Einzelfallakten insb. zum Ruhen der Rente wegen staatsfeindlicher Betätigung (R 89/22702-22707 bzw. 23084-23087).

Von der RfA selbst bzw. der Nachfolgebehörde BfA wurden nur wenige Akten an das Bundesarchiv abgegeben, so dass der Großteil noch im sogenannten RfA-Archiv bei der DRV-Bund in der Ruhrstraße liegt. Der dortige Bestand umfasst wie erwähnt insgesamt ca. 500 Akteneinheiten oder ca. 60 lfd. Meter mit Laufzeit 1919 bis 1945 (vereinzelt auch darüber hinausgehend bzw. weiter zurückreichend), klarer Schwerpunkt ist aber die NS-Zeit. Der Bestand ist wiederum in zwei unterschiedlich geordnete Teilbestände aufgeteilt. Der erste steht in Aktenschränken und ist mit Signaturen versehen (von Nr. 1 bis Nr. 233), teils nicht nummeriert.. Hier finden sich Überlieferungen zur Organisation der Behörde und ihrem operativen Geschäft bzw. der Verwaltungstätigkeit (allein zehn Akten zu Rentenverfahren der Abteilung I Leistung, Abteilungsverfügungen, Präsidialverfügungen, Geschäftsverteilungspläne, Entscheidungssammlungen und Schriftwechsel mit dem RVA und RAM sowie diversen weiteren Stellen wie DAF und Landesversicherungsanstalten, Protokolle von Direktoriums- und Beiratssitzungen, Stellungnahmen zu den laufenden und geplanten gesetzlichen Maßnahmen, Statistik sowie die Einführung rentenversicherungsrechtlicher Maßnahmen in den besetzten bzw. eingegliederten Gebieten wie Österreich, Polen, Sudetenland). Der zweite Teilbestand umfasst ca. 800 Akten zu zahlreichen versicherungsrechtlichen Einzelproblemen (Generalakten), die in 126 Fächern liegen. Hierzu existiert ein alter, 108-seitiger Aktenplan nach Stichworten und zugeordneten Fachnummern. In allen diesen Akten finden sich hunderte von Briefen und Eingaben der Versicherten selbst an die RfA und die entsprechenden Antwortschreiben der Behörde, so dass nicht nur die Perspektive der RfA, sondern auch diejenige der Versicherten deutlich widergespiegelt wird. Auffällig ist jedoch, dass in dem Bestand des RfA-Archivs keine Akten zu Heilverfahren/Gesundheitsfürsorge (Abt. II) überliefert sind und auch nichts zum sozialen Wohnungsbau/Gagfah zu finden ist. Den Krieg überstanden hatten zunächst auch sämtliche Personalakten, die jedoch nicht im RfA-Archiv abgelegt worden waren. Zu Beginn der 1990er Jahre wurde dieser wertvolle Bestand jedoch vollständig vernichtet.

\section{DRV-Bund, Berlin}

„RfA-Archiv“, Berlin (Verzeichnis wichtiger benutzter Aktengruppen/Kurzrepertorium)

\section{a Verwaltungsorganisation}

RfA Nr. 7: Besprechungen Abt. I Leistung 1931-1941

RfA Nr. 18-27: Schriftwechsel Abt. I Leistung Allgemein 1933-1943/44

RfA Nr. 72-77: Geschäftsverteilung der Abt. I Leistung

Ә OpenAccess. (c) 2019 Paul Erker, publiziert von De Gruyter. (cc)BBY-NC-ND Dieses Werk ist lizenziert unter der Creative Commons Attribution-NonCommercial-NoDerivatives 4.0. https://doi.org/10.1515/9783110652741-011 
RfA Nr. 90-98: Schriftwechsel der Abt. I Leistung mit Arbeitgebern/Arbeitnehmern 1931 bis 1945 RfA Nr. 205-206: Geschäftsgang der Abt. I Leistung

RfA o. Sign., 2 Ordner: Rundschreiben an die Überwachungsbeamten 1939-1945

RfA Nr. 1-3: Präsidialverfügungen 1924 bis 1944

RfA, Fach 1, Nr. 1-4: Abteilungsverfügungen 1937-1944

RfA-Archiv, o. Signatur (Regal 5): Abteilungsverfügungen 1921-1942

BA/R89-3468 bis 3470: Protokolle der Beiratssitzungen 1 (25.11.1935 bis 16.4.1943)

RfA Nr. 78 und R 89/3468, R 89/3470: Geschäftsberichte RfA 1935 bis 1938 und 1939 bis 1942.

\section{b RV in den eingegliederten und besetzten Gebieten}

RfA Nr. 196, Nr. 196 a: Eingegliederte Gebiete/Sudetenland

RfA Nr. 169, Nr. 170-174 und 176, 177-180: RV Sudetenland und Protektorat

RfA Nr. 68 und Nr. 69, 69 a, 69 b: Einführung RV in Österreich, Sudetenland, Protektorat

RfA, Fach 34, Nr. 3-7: Generalgouvernement/Ostgebiete/Polen

Fach 119, Nr. 1: dito

RfA Nr. 162-163 a-c: Ostgebiete/Polen

RfA Nr. 165 und 165a, Nr. 166, 166 a-e: Ostgebiete/Polen

RfA Nr. 138, Nr. 139

RV in Luxemburg

RfA Nr. 186, 187, Nr. 195 a: RV im Saargebiet

RfA Nr. 130, Nr. 131, Nr. 131 a, Nr. 132, 132 a, Nr. 133, 133 a: RV in Elsass/Lothringen

\section{c Statistische Unterlagen}

RfA Nr. 202: Diverse Statistiken

RfA Nr. 66: Zahlungen des Reichs zur RV 1941-1943

RfA-Archiv, Handakte Direktor Granzow, Statistische Sonderhefte der RfA Nr. 1 (1935) bis Nr. 8 (1939)

Fach 13, Nr. 1: Beitragsstatistik

RfA Nr. 200: Kapitalisierung/Finanzierung der RV (Verhältnis IV und RV 1943/44)

Fach 101, Nr. 6-9: div. Monatsberichte 1933-1944/45

RfA Nr. 10 und Nr. 11: Bilanzen

RfA Nr. 79 bis Nr. 81: Statistik/Bilanzen

\section{d Die einzelnen RV-Gesetze und Vo und deren Umsetzung}

RfA-Archiv, Handakte Direktor Granzow, Gesetzeskommentierungen, Stellungnahmen

Fach 108, Nr. 1-17: Gesetzesänderungen

Fach 109, Nr. 1-19: Gesetzesänderungen

Fach 110, Nr. 1-17: Gesetzessänderungen

Fach 111, Nr. 1-12: Gesetzesänderungen

Fach 112, Nr. 1-9: Gesetzesänderungen

Fach 113, Nr. 1-18: Gesetzesänderungen

Fach 114, Nr. 1-16: Gesetzesänderungen

RfA Nr. 37: VO über Anpassung der RV an totalen Kriegseinsatz 1944 
RfA Nr. 67: VO über RV während des besonderen Einsatzes der Wehrmacht vom 13.10.1939

RfA Nr. 35: Auslegung VO vom 1.10.1942

RfA Nr. 36: VO betr. Leistungsverbesserung in besetzten Gebieten 12.10.1943

Fach 55, Nr. 3 und Nr. 4: Kürzungen und Ruhen der Renten aus vorherigen Gesetzen 1933/1934

RfA Nr. 28: Handwerker-Gesetz

RfA Nr. 29: Leistungsverbesserungsgesetz

RfA Nr. 30: KV der Rentner $1941 \mathrm{ff}$.

RfA Nr. 31: Gesetz vom 19.6.1942

RfA Nr. 58:Debatten über Gesetz vom 17.5.1934/Durchführung

\section{e RV-rechtliche Detailprobleme:}

RfA Nr. 56, Nr. 57: Wanderversicherte

Fach 96 und Fach 97, Nr. 1-5: Ersatzzeiten WK II/Wehrmacht (Steigerungsbeträge)

Fach 22, 1-12: Entgelt (Anrechnung für SV-Beiträge)

Fach 94, Nr. 1-5: u. a. betr. freiwillige Weiterversicherung

Fach 93, Nr. 1-4: Wartezeit §53, 1932-1944

Fach 78-89: betr. Versicherungspflicht einzelner Berufsgruppen (Schriftwechsel)

Fach 11, Nr. 1-3 sowie Fach 15, Nr. 1-4: Beitragsverfahren (intus: Berichte von

Überwachungsbeamten)

RfA Nr. 101 bis Nr. 109: RVA-Entscheidungen/Berufungsverfahren/Klagen gegen die RfA 1923-1945

\section{f Betr. Juden und RV im Ausland}

RfA Nr. 167 und Nr. 168

RfA Nr. 88, Nr. 126 und Nr. 127, Nr. 197 und Nr. 198: Juden/Devisen; Rentenzahlungen Ausland.

RfA Nr. 210, 210a, Nr. 214: Rentenzahlungen ins Ausland

\section{g Betr. RfA und DAF}

Fach 116, Nr. 9: Rechtshilfe DAF

Fach 119, Nr. 2: Altersversorgungswerk DAF

Fach 19, Nr. 2-3: DAF

RfA Nr. 22: DAF

RfA Nr. 86, Nr. 87: DAF

\section{Registratur der Grundsatzabteilung sowie weiterer Abteilungen}

\section{Bundesarchiv („BArch“) Berlin,}

Bestand R 89: Reichsversicherungsamt

Bestand R 112: RfA

Bestand BArch NS 18/714: Reichsbund der deutschen Kapital- und Kleinrentner 
Bestand R 3901 (Reichsarbeitsministerium), 103525, Bd. 1-3 (Personalakte Grießmeyer) Bestand R 2/18562 (Reichsfinanzministerium)

BA/9361-I (OPG) Parteigerichtsverfahren gegen Grießmeyer

\section{Privatarchiv Dr. Hans-Jörg Bonz}

Hans-Jörg Bonz, langjähriger Ministerialdirektor im Bundesarbeitsministerium bzw. Sächsischen Sozialministerium wurde 1988 mit der Erarbeitung einer Gesamtgeschichte der Rentenversicherung bzw. RfA/BfA beauftragt und dafür drei Jahre freigestellt. Aus dieser Zeit verfügt Bonz noch über ein Privatarchiv an damals gefertigten Aktenkopien (keine Originale), die in 25 Akten Unterlagen vor allem aus dem damaligen „RfA-Bestand“ bei der BfA enthalten, die inzwischen zum Teil verloren gegangen sind. Darunter sind insb. zwei Ordner mit inzwischen vernichteten oder unauffindbaren Personalakten für die Zeit von 1933-1945. Daneben ein Ordner Mitteilungen der RfA, ein Ordner Verfügungen der Leistungs- und Versicherungsabteilung 1925 bis 1943; ein Ordner RfA, Aufgaben und Tätigkeit, ein Ordner Treuhandverwaltung, UAB/RfA-Presseberichte 1945 bis 1948; ein Ordner Verwaltungsratssitzungen 1913 bis 1922.

Die Geschichte der Angestelltenversicherung, unveröffentl. Manuskript von 1988, zwei Ordner insg. 652 Seiten.

\section{Gedruckte Quellen (überwiegend aus dem Bestand der Bibliothek der DRV-Bund, Ruhrstraße)}

25 Jahre Angestelltenversicherung 1913-1937, hrgg. von der RfA, Berlin 1937.

Angestelltenversicherungsgesetz in der Fassung des Ausbaugesetzes v. 21. Dez. 1937 nebst Ausführungsbestimmungen: Kommentar, Verfasser: Allendorff, Franz und Fritz Haueisen; Berlin 1938.

Bericht der Deutschen Revisions- und Treuhand-Aktiengesellschaft Berlin über die bei der Reichsversicherungsanstalt für Angestellte vorgenommene Prüfung der vom 1.7.1937 bis 30.6.1938 zugesagten Hypothekendarlehen.

Bericht der Deutschen Revisions- und Treuhand-Aktiengesellschaft Berlin über die bei der Reichsversicherungsanstalt für Angestellte vorgenommene Prüfung der Kassengebahrung, der Buchführung und des Rechnungsabschlusses für das Wirtschaftsjahr 1936.

Bericht des Direktoriums (ab 1935 Geschäftsbericht des Leiters/Präsidenten) der Reichsversicherungsanstalt für Angestellte über das Geschäftsjahr 1913-1938, Berlin 1914-1939 [RD 91/2].

Bericht über die Arbeiten des Zweiten Internationalen Kongresses der Sozialversicherungsfachleute in Dresden vom 4.-8.9.1936, hrgg. vom Reichsverband Deutscher Landesversicherungsanstalten, Stuttgart/Berlin 1936.

Bericht über die Arbeiten des Dritten Internationalen Kongresses der Sozialversicherungsfachleute in Wien vom 18.-22.5.1938, hrgg. vom Reichsverband der Sozialversicherungsträger in Wien, Wien 1938.

Bericht über die Geschäftsprüfung durch das RVA bei der Reichsversicherungsanstalt für Angestellte in der Zeit vom Oktober 1938 bis März 1939 (104 Seiten).

Bericht und Anhang der Deutschen Revisions- und Treuhand-Aktiengesellschaft Berlin über die bei der Reichsversicherungsanstalt für Angestellte vorgenommene Prüfung der Organisation der einzelnen Geschäftsabteilungen, Berlin 31. Oktober 1931. 
DAF-Entscheidungssammlung. Entscheidungen aus dem Arbeitsrecht und Sozialversicherungsrecht, hrgg. vom Amt für Rechtsberatungsstellen im Zentralbüro der Deutschen Arbeitsfront, Berlin 1937.

Das Angestelltenversicherungsgesetz, mit sämtlichen Ausführungsbestimmungen und allen sonstigen die Angestelltenversicherung berührenden Gesetzen, Verordnungen, Bekanntmachungen und zwischenstaatlichen Verträgen, nach dem neuesten Stande (1. März 1938-Okt. 1939), Kommentar von Hugo Koch und Otto K. Hartmann, Berlin 1939.

Das Fremdrenten- und Auslandsrenten-Neuregelungsgesetz vom 25.2.1960 in der praktischen Anwendung, Schriftenreihe der BfA Nr. 15, Berlin 1960.

Denkschrift über die voraussichtliche Entwicklung der Vermögenslage der Reichsversicherungsanstalt für Angestellte, Berlin 1940.

Der RfA-Beamte. Zeitschrift des Verbandes der Beamten der Reichsversicherungsanstalt für Angestellte, Berlin 1928-1933.

Der Überwachungsdienst der RfA (3 S.), Berlin 1934.

Deutsche Rentenversicherung, hrgg. vom Reichsverband Deutscher Rentenversicherungsträger 11 (1939)-15 (1943).

Die Beitragsnachweise der Angestelltenversicherung. Teil 1: Ein Überblick über die Beitragsentrichtung zur Angestelltenversicherung und die Kontenführung bei der RfA/BfA, Schriftenreihe der BfA Nr. 16, Berlin 1964.

Die Rationalisierung der Sozialversicherung: Die Arbeiten des ersten Internationalen Kongresses der Sozialversicherungsfachleute, Budapest 1935.

Dobbernack, Wilhelm, Einführung der Reichsversicherung in den eingegliederten Ostgebieten (16 S.), Berlin 1942.

Gagfah 1918 -1968. Eine Dokumentation, Hamburg 1968.

Grundsätze für die Ausbildung und Beschäftigung im einfachen mittleren Dienst bei der Reichsversicherungsanstalt für Angestellte, Berlin 1937.

Mischkowsky, Heinz-Georg, Die eingegliederten Ostgebiete und das Generalgouvernement in ihrer Bedeutung für die Dienststelle I Allgemein, unter besonderer Berücksichtigung der Staatszugehörigkeitsverhältnisse in diesen Gebieten (nur für den Dienstgebrauch), Berlin 1951.

Mitteilungen der Reichsversicherungsanstalt für Angestellte, herausgegeben von der Reichsversicherungsanstalt für Angestellte, Berlin 1923-1945 [RD 91/4= Jahrgänge 1939-1944].

Monatshefte für NS-Sozialpolitik 1 (1933)-11 (1943).

Monatsschrift für Arbeiter- und Angestellten-Versicherung 20 (1933)-31 (1943).

Pin, Josef Otto, Die Umsiedlungsaktionen 1939-1945 in ihrer Bedeutung für die Sozialversicherung, Berlin 1959 (MS 203 Seiten); nur für den Dienstgebrauch innerhalb der BfA.

Rechnungsabschluss der RfA für die Zeit vom 1. Januar 1944 bis 8. Mai 1945, Berlin 1951 (Sign. II A $3 c)$.

Richtlinien für die Laufbahn der Anwärter des gehobenen mittleren Dienstes bei der Reichsversicherungsanstalt für Angestellte, Berlin 1938.

Selbstverwaltung in der Angestelltenversicherung vor 1945 (zusammengestellt von der Bibliothek der BfA), Berlin 1958.

Soziale Praxis. Zeitschrift für Aktienwesen, Gesellschaftsrecht und Sozialpolitik 42 (1933)-52 (1943) (1944 wurden die Zeitschriften Soziale Praxis und Monatshefte für NS-Sozialpolitik zusammengelegt unter dem Titel Die deutsche Sozialpolitik).

Statistisches Sonderheft der RfA Nr. 1-8 (1935-1939).

Treuhandverwaltung der stillgelegten Sozialversicherungsträger, RfA, Verwaltungsbericht 1945-1952, (erstattet anlässlich des 40-jährigen Bestehens der Angestelltenversicherung), Berlin 1952. 
Was muss jeder von der Angestellten-Versicherung (Rentenversicherung der Angestellten) wissen?

Eine leichtverständliche Darstellung und Erläuterung nach dem neuesten Stande unter

Berücksichtigung der neuen Kriegsgesetze und Verordnungen, Essen 1942 (39 Seiten).

Wolkersdörfer, Hans, Sozialpolitisches Lexikon, Berlin 1938.

\section{Aufsätze und Reden von RfA-Präsident Albert Grießmeyer}

Angestelltenversicherung und Wirtschaftskrise, in: Hamburger Fremdenblatt vom 16.9.1931.

Rede Grießmeyers auf der Bezirkstagung der Ortsausschüsse der Vertrauensmänner der

Angestelltenversicherung für Württemberg und Hohenzollern in Stuttgart am 8.5.1932, in:

Selbstverwaltung in der Angestelltenversicherung vor 1945 (zusammengestellt von der

Bibliothek der BfA), Berlin 1958.

Die Angestelltenversicherung im Jahre 1934, in: Der Sozialversicherungsbeamte 3 (1935),

S. $178-179$.

Das Jahr 1935 bei der Angestelltenversicherung, in: Der Sozialversicherungsbeamte 4 (1936),

S. $252-253$.

Die Berechtigung und Bedeutung der Angestelltenversicherung in Deutschland, in: Le Assicurazioni Soziali 12 (1936), S. 178-183.

Vortrag über „Die Sanierung der Rentenversicherungen“ auf der Sondertagung der

Reichsfachgruppe Sozialversicherung am 15.10.1937 in Frankfurt, nur Bericht dazu in: Soziale

Praxis 46 (1937) sowie in: Deutsche Invalidenversicherung 9 (1937), S. 225-226.

Geleitwort zur Festschrift: 25 Jahre Angestelltenversicherung 1913-1937.

Rede am 17.1.1938 anlässlich Feierstunde zum 25-jährigen Bestehen der RfA, in:

RfA-Geschäftsbericht 1937, S. 41- 47.

Die Leistungen der Reichsversicherung für Angestellte, in: VB vom 7.1.1938.

Ausführlicher Diskussionsbeitrag zur Vortragsreihe „Vermögensanlage der

Sozialversicherungsträger" auf dem Dritten Internationalen Kongress der

Sozialversicherungsfachleute in Wien vom 18. bis 22.5.1938, in: Bericht über die Arbeiten des

Dritten Internationalen Kongresses der Sozialversicherungsfachleute in Wien vom 18.-

22.5.1938, hrgg. vom Reichsverband der Sozialversicherungsträger in Wien, Wien 1938,

S. $202-207$.

Wesen und Umfang der Deutschen Angestelltenversicherung, in: Le Assicurazioni Soziali 15 (1939),

S. $492-498$.

Die Altersversorgung des Handwerksmeisters (zusammen mit Felix Schüler), Berlin 1939 (47 Seiten).

Rede (Lagebericht RfA) jeweils auf den RfA-Beiratssitzungen am 17.4.1939, 11.12.1939, 8.4.1940,

2.12.1940, 20.4.1942. in: Niederschriften der Beiratssitzungen BArch R 89/3470.

Gefolgschaftsversorgung bei der sozialen Rentenversicherung, in: Die Deutsche Volkswirtschaft, Januar 1939, S. 40-42.

Betrachtungen zur Altersversorgung für das Deutsche Handwerk, in: Deutsche Rentenversicherung 11 (1939), Nr. 2, S. $18-20$.

Angestelltenversicherungsgesetz in der Fassung des Ausbaugesetzes v. 21. Dez. 1937 nebst

Ausführungsbestimmungen: Kommentar, Verfasser: Allendorff, Franz und Fritz Haueisen; Mit einem Geleitwort v. Albert Grießmeyer, Berlin 1938 (511 Seiten).

Das Angestelltenversicherungsgesetz, mit sämtlichen Ausführungsbestimmungen und allen sonstigen die Angestelltenversicherung berührenden Gesetzen, Verordnungen, Bekanntmachungen und zwischenstaatlichen Verträgen, nach dem neuesten Stande (1. März 1938- Okt. 1939) Kommentar von Hugo Koch und Otto K. Hartmann, mit einem Geleitwort von Albert Grießmeyer, Berlin 1939.

Die Altersversorgung des Deutschen Volkes, in: Le Assicurazioni Soziali 16 (1940), S. 621-625. 
Die Angestelltenversicherung im werdenden Großdeutschland, in: Amtliche Nachrichten für die Reichsversicherung 22, 1942, S. 256-262.

Das Angestelltenversicherungsgesetz: nach dem neuesten Stande (Mai 1942-April 1943) mit sämtlichen Ausführungsbestimmungen, Kommentar, Hrsg./Verfasser: Hugo Koch, Otto K. Hartmann, mit einem Geleitwort von Albert Grießmeyer, Berlin 1943 (789 Seiten).

Ansprache anlässlich der Einführung von Präsident Schmitt durch den Reichsarbeitsminister am 26.7.1944 im RVA (in: Deutsche Rentenversicherung 16 (1944), S. 30).

\section{Aufsätze/Publikationen anderer RfA-Angehöriger:}

A. von Altrock, Das Beitragsstreitverfahren der Rentenversicherung, in: Deutsche Rentenversicherung 16 (1944), S. 5-10.

A. von Altrock, Das Problem der Altersgrenze in der Angestelltenversicherung, in: Deutsche Rentenversicherung 10(?) (1941), S. 132-134.

Regierungsrat Dr. Haueisen, Die Reichsversicherungsanstalt für Angestellte als Trägerin von Gemeinschaftsaufgaben, in: Deutsche Rentenversicherung 11 (1939), S. 124-130, 168-169.

Otto Hartmann, Zur neuen Beitragserstattung in der Rentenversicherung der Arbeiter und Angestellten, in: Deutsche Rentenversicherung 11 (1939), S. 23-26.

Hugo Koch, Geschäftsgang und Verfahren der Abteilung I Leistung. (Zusammenstellung der Verfügungen, Referentenbesprechungen und der allgemeinen Grundsätze über das Verfahren nach dem Stand vom 1. April 1935 unter Berücksichtigung der Verwaltungsübung und Rechtsprechung), gedruckte Broschüre, 73 Seiten.

Curt Perlin, Handwörterbuch der Angestelltenversicherung. Rentenversicherung der Angestellten, Berlin/Essen 1939.

Curt Perlin, Die Rentenversicherung im Kampf gegen die Tuberkulose, in: Monatshefte für NS-Sozialpolitik 6 (1939), S. $456-459$.

R. Wegener, Ruhen, Kürzung und Einstellung der Rentenzahlung der Angestelltenversicherung an Beispielen erläutert, Berlin 1936.

\section{Literaturverzeichnis}

50 Jahre Angestelltenversicherung, hrgg. von der BfA, Berlin 1963.

Alterssicherung in Deutschland. Festschrift für Franz Ruland zum 65. Geburtstag, hrgg. von Ulrich Becker, Franz-Xaver Kaufmann, Bernd Baron von Maydell und Winfried Schmähl, Baden-Baden 2007.

Aly, Götz, Hitlers Volksstaat. Raub, Rassenkrieg und nationaler Sozialismus, Frankfurt 2005.

Aye, Adolf, Die Selbstverwaltung in der deutschen Sozialversicherung, Weissenburg 1950.

Bonz, Hans-Jörg, Die Stellung der Juden in der Deutschen Rentenversicherung, in: Zeitschrift für Sozialreform 34 (1988), H. 7, S. 425-427.

Bonz, Hans-Jörg, Für Staatsfeinde keine Rente. Das Ruhen der Renten bei staatsfeindlicher Betätigung im nationalsozialistischen Deutschland, in: Zeitschrift für Sozialreform,37 (1991), H. 9, S. $517-531$.

Bonz, Hans-Jörg, Geplant, aber nicht in Kraft gesetzt: Das Sonderrecht für Juden und Zigeuner in der Sozialversicherung des nationalsozialistischen Deutschland, in: Zeitschrift für Sozialreform 38 (1992), H. 3, S. 148-164.

Conrad, Christoph, Alterssicherung, in: Hans Günter Hockerts (Hrsg.), Drei Wege deutscher Sozialstaatlichkeit. München 1998, S. 101-116. 
Eden, Sören, Marx, Henry, Schulz, Ulrike, Ganz normale Verwaltungen? Methodische Überlegungen zum Verhältnis von Individuum und Organisation am Beispiel des Reichsarbeitsministeriums 1919 bis 1945, in: VfZ 66 (2018), S. 487-520.

Eichenhofer, Eberhardt, Rische, Herbert, Schmähl, Winfried (Hrsg.), Handbuch der gesetzlichen Rentenversicherung, Köln 2011.

Geyer, Martin H., Soziale Rechte im Sozialstaat: Wiederaufbau, Krise und konservative Stabilisierung der deutschen Rentenversicherung 1924-1937, in: Klaus Tenfelde (Hrsg.), Arbeiter im 20. Jhdt, 1991, S. 406-434.

Glootz, Tanja Anette, Geschichte der Angestelltenversicherung des 20. Jahrhunderts, Berlin 1999. Hachtmann, Rüdiger, Elastisch, dynamisch und von katastrophaler Effizienz. Zur Struktur der Neuen Staatlichkeit des Nationalsozialismus, in: Sven Reichardt, Wolfgang Seibel (Hrsg.), Der prekäre Staat. Herrschen und Verwalten im Nationalsozialismus, Frankfurt 2011, S. 29-73.

Hachtmann, Rüdiger, Reichsarbeitsministerium und Deutsche Arbeitsfront. Dauerkonflikt und informelle Kooperation, in: Alexander Nützenadel (Hrsg.), Das Reichsarbeitsministerium im Nationalsozialismus. Verwaltung, Politik, Verbrechen, Göttingen 2017, S. 137-173.

Heess, W., Fetscher S., Die Kriminalität auf dem Gebiete der Invalidenversicherung und ihre Bekämpfung. In: Archiv für Kriminologie 102 (1938), S. 1-38.

Hockerts, Hans Günter, Sicherung im Alter. Kontinuität und Wandel der gesetzlichen Rentenversicherung 1889-1979, in: Werner Conze, M. Rainer Lepsius (Hrsg.), Sozialgeschichte der Bundesrepublik Deutschland. Beiträge zum Kontinuitätsproblem, Stuttgart 1983, S. $296-323$.

Hockerts, Hans Günter, Sozialpolitische Entscheidungen im Nachkriegsdeutschland, Stuttgart 1980.

Hockerts, Hans Günter, Vom Wohlfahrtsstaat zum Wohlfahrtsmarkt? Privatisierungstendenzen im deutschen Sozialstaat, in: Nobert Frei, Dietmar Süß (Hrsg.), Privatisierung. Idee und Praxis seit den 1970er Jahren, Weimar 2012, S. 70-87.

Kirchberger, Petra, Die Stellung der Juden in der deutschen Rentenversicherung, in: Götz Aly u.a. (Hrsg.), Sozialpolitik und Judenvernichtung. Gibt es eine Ökonomie der Endlösung?, Berlin 1987, S. 111-132.

Klimo, Alexander, Rentenversicherung zwischen Arbeitseinsatz und Diskriminierung, in: Alexander Nützenadel (Hrsg.), Das Reichsarbeitsministerium im Nationalsozialismus. Verwaltung, Politik, Verbrechen, Göttingen 2017, S. 214-245.

Klimo, Alexander, Im Dienste des Arbeitseinsatzes. Rentenversicherungspolitik im „Dritten Reich“, Göttingen 2018.

Köhler, Peter A., Entwicklungslinien der 100jährigen Geschichte der gesetzlichen Rentenversicherung: Die Zeit von 1891-1957, in: Ruland, Franz (Hrsg.), Handbuch der gesetzlichen Rentenversicherung: Festschrift aus Anlass des 100jährigen Bestehens der gesetzlichen Rentenversicherung, im Auftr. d. Vorstandes d. Verb. Dt. Rentenversicherungsträger (VDR), Frankfurt 1990, S. 51-92.

Mason, Timothy W., Sozialpolitik im Dritten Reich. Arbeiterklasse und Volksgemeinschaft, Opladen 1977.

Mentel, Christian, Weise, Nils, Die Zentralen Deutschen Behörden und der Nationalsozialismus. Stand und Perspektiven der Forschung, hrgg. von ZZF und IfZ, Berlin/München 2016, als PDF unter: https://www.ifz-muenchen.de/fileadmin/user_upload/Neuigkeiten\% 202016/2016_02_13_ZZF_IfZ_PM_BKM-Studie_FINAL_Neu.pdf.

Mierzejewski, Alfred C., A History of the German Public Pension System. Continuity and Change, N.Y./London 2016.

Miquel, Marc von (Hrsg.), Sozialversicherung in Diktatur und Demokratie, Essen 2007.

Möckel, Benjamin, „Nutzlose Volksgenossen“? Der Arbeitseinsatz alter Menschen im Nationalsozialismus. Eine kulturhistorische und sozialgeschichtliche Untersuchung über den Altersdiskurs und die Sozialpolitik des Alters im Nationalsozialismus, Berlin 2010. 
Mörschel, Richard, Die Finanzierungsverfahren in der Geschichte der gesetzlichen Rentenversicherung, in: Deutsche Rentenversicherung (1990), S. 619-661.

Nickl, Walter, Die Selbstverwaltung der Versicherungsträger der Reichsversicherung, Diss. Erlangen 1938.

Nitsche, Michael, Die Geschichte des Leistungs- und Beitragsrechts der gesetzlichen Rentenversicherung von 1889 bis zum Beginn der Rentenreform, Frankfurt 1986.

Nützenadel, Alexander (Hrsg.), Das Reichsarbeitsministerium im Nationalsozialismus. Verwaltung, Politik, Verbrechen, Göttingen 2017.

Pawlita, Cornelius, Rentenversicherungsrechtliche Aspekte verfolgungsbedingter Zwangsarbeit im Nationalsozialismus, in: Zeitschrift für Sozialreform 44 (1998), S. 1-21.

Recker, Marie-Luise, Nationalsozialistische Sozialpolitik im Zweiten Weltkrieg. München 1985.

Reichardt, Sven, Seibel, Wolfgang, Radikalität und Stabilität: Herrschen und Verwalten im Nationalsozialismus, in: dies. (Hrsg.), Der prekäre Staat. Herrschen und Verwalten im Nationalsozialismus, Frankfurt 2011, S. 7-27.

Reidegeld, Eckart, Staatliche Sozialpolitik in Deutschland, Bd. II: Sozialpolitik in Demokratie und Diktatur 1919-1945, Wiesbaden 2006, insbesondere S. 441-561.

Ruland, Franz, Die Geschichte des Verbandes Deutscher Rentenversicherungsträger (VDR), in: Deutsche Rentenversicherung 60 (2005), S. 354-361.

Rücker, Simone, Das Rechtsberatungswesen von 1919-1945 und die Entstehung des Rechtsberatungsmissbrauchsgesetz von 1935, Tübingen 2007.

Sachße, Christoph, Tennstedt, Florian, Der Wohlfahrtsstaat im Nationalsozialismus, in: Zeitschrift für Sozialreform 38 (1992), S. 129-148.

Schäfer, D.-J., 75 Jahre Verband Deutscher Rentenversicherungsträger, in: Deutsche Rentenversicherung 49 (1994), S. 571-636.

Scharf, Gerhard, Die Entwicklung der Angestelltenversicherung in Zahlen, in: Die Angestelltenversicherung 3 (1956), S. 269-276.

Schlegel-Voß, Lil-Christine, Alter in der „Volksgemeinschaft“. Zur Lebenslage der älteren Generation im Nationalsozialismus, Berlin 2005.

Schmiechen-Ackermann, Detlef u. a. (Hrsg.), Der Ort der „Volksgemeinschaft“ in der deutschen Gesellschaftsgeschichte, Paderborn 2017.

Seibel, Wolfgang, Verwaltung verstehen. Eine theoriegeschichtliche Einführung, Frankfurt 2016.

Stolleis, Michael, Historische Grundlagen. Sozialpolitik in Deutschland bis 1945, in: Geschichte der Sozialpolitik in Deutschland seit 1945, hrgg. vom Bundesministerium für Arbeit und Sozialordnung und vom Bundesarchiv. Bd. 1: Grundlagen der Sozialpolitik, Baden-Baden 2001, S. 199-332, hier S. 321f.

Süß, Winfried, Die sozialdemokratische Sehnsucht nach Sicherheit. Ernst Schellenberg und die Sozialpolitik der Bonner Republik, in: Theresia Bauer u.a. (Hrsg.), Gesichter der Zeitgeschichte. Deutsche Lebensläufe im 20. Jahrhundert, München 2009, S. 297-314.

Tálos, Emmerich, Sozialpolitik in der „Ostmark“. Angleichungen und Konsequenzen, in: ders. u.a. (Hrsg.), NS-Herrschaft in Österreich. Ein Handbuch, Wien 2001, S. 376-408.

Teppe, Karl, Zur Sozialpolitik des Dritten Reiches am Beispiel der Sozialversicherung, in: AfS 17 (1977), S. 195-250.

Wehner, Christoph Die Landesversicherungsanstalten Baden und Württemberg im „Dritten Reich“. Personalpolitik, Verwaltung und Rentenpraxis 1933-1945, Bochum 2017. 